\title{
Crohn Disease
}

National Cancer Institute

\section{Source}

National Cancer Institute. Crohn Disease. NCI Thesaurus. Code C2965.

A gastrointestinal disorder characterized by chronic inflammation involving all layers of the intestinal wall, noncaseating granulomas affecting the intestinal wall and regional lymph nodes, and transmural fibrosis. Crohn disease most commonly involves the terminal ileum; the colon is the second most common site of involvement. 